\title{
A study on regional differences in decidualization of the mouse uterus
}

\author{
Miao Zhao, Wen-Qian Zhang and Ji-Long Liu \\ College of Veterinary Medicine, South China Agricultural University, Guangzhou, China \\ Correspondence should be addressed to J-L Liu; Email: jilongliu@scau.edu.cn
}

\begin{abstract}
Although regional differences in mouse decidualization have been recognized for decades, the molecular mechanisms remain understudied. In the present study, by using RNA-seq, we compared transcriptomic differences between the anti-mesometrial (AM) region and the mesometrial $(\mathrm{M})$ region of mouse uterus on day 8 of pregnancy. A total of 1423 differentially expressed genes were identified, of which 811 genes were upregulated and 612 genes were downregulated in the AM region compared to those in the $M$ region. Gene ontology analysis showed that upregulated genes were generally involved in cell metabolism and differentiation, whereas downregulated genes were associated with lymphocyte themes and immune response. Through network analysis, we identified a total of 6 hub genes. These hub genes are likely more important than other genes due to their key positions in the network. We also examined the promoter regions of differentially expressed genes for the enrichment of transcription factor-binding sites. In the end, we demonstrated that a similar regional gene expression pattern can be observed in the artificial decidualization model. Our study contributes to an increase in the knowledge on the molecular mechanisms underlying regional decidualization in mice.

Reproduction (2017) 153 645-653
\end{abstract}

\section{Introduction}

One of the essential steps for the establishment and maintenance of human pregnancy is the decidualization of endometrial stromal cells (Wang \& Dey 2006). During decidualization, stromal cells undergo proliferation and subsequent differentiation into large epithelioid cells characterized by the secretion of decidual prolactin (PRL) and insulin-like growth factor-binding protein 1 (IGFBP1) (Telgmann \& Gellersen 1998). Decidualization has various functional roles, such as the regulation of embryo implantation, modulation of maternal immune response to embryonic antigens and control of placentation (Blois et al. 2011, Barrientos et al. 2014). Defects in decidualization during early pregnancy may lead to severe pregnancy complications, such as pregnancy loss, intrauterine growth restriction and preeclampsia (Gellersen \& Brosens 2014).

The mouse is widely used as an animal model for the study of human pregnancy. Human decidualization initiates spontaneously in the secretory phase of menstrual cycle in response to steroid hormones. Mouse decidualization is slightly different in that it is an embryo-dependent process (Ramathal et al. 2010). Mouse decidualization occurs in a temporal and regional fashion (Dey et al. 2004). Shortly after embryo implantation, stromal cells adjacent to the implanting embryo in the anti-mesometrial (AM) region of the uterus show the first sign of decidualization reaction, which form the primary decidual zone (PDZ). Decidualization then spreads rapidly throughout the AM region, forming the secondary decidual zone (SDZ). Meanwhile, stromal cells in the mesometrial $(\mathrm{M})$ region of uterus begin to undergo decidualization to form the mesometrial decidual area. The decidualized cells in the AM region and the $\mathrm{M}$ region are morphologically different. The AM region is a compact tissue with a portion of polyploid cells, whereas the $M$ region is not a compact tissue and polyploidy is absent. From a functional perspective, decidualization in the AM region is involved in the accommodation of embryo implantation and decidualization in the $M$ region contributes to the preparation of placentation.

However, the molecular information about regional differences in decidualization currently remains unavailable. In the present study, we used RNA-seq approach to investigate the gene expression profiles for the $A M$ region and the $M$ region of mouse uterus on day 8 of pregnancy. Through bioinformatic analysis of the data, our study contributes to an increase in the knowledge on the molecular mechanisms underlying regional decidualization in mice. 


\section{Materials and methods}

\section{Sample collection}

Natural pregnancy model was established using CD-1 mice by co-caging adult females with fertile males. The day of the vaginal plug was designated as day 1 of pregnancy. The uterine segments of implantation sites were obtained on day 8 of pregnancy $(n=3)$. Embryonic tissues were removed under a stereomicroscope. A complete removal was confirmed in sequential frozen sections. The embryo-free uterine segments were cut into halves to separate the mesometrial side (M) and the anti-mesometrial side (AM) of uterus. In addition to natural pregnancy, an artificial decidualization model was also established. In this model, female mice were mated with vasectomized males to induce pseudo-pregnancy. Intrauterine injection of $10 \mu \mathrm{L}$ sesame oil was performed to mimic the stimulation of the embryo on day 4 of pseudo-pregnancy. Uterine samples were collected on day 8 of pseudo-pregnancy $(n=3)$. All collected samples were then snap-frozen in liquid nitrogen and stored at $-80^{\circ} \mathrm{C}$ until use. All animal procedures were approved by the Institutional Animal Care and Use Committee of South China Agricultural University.

\section{RNA-seq}

Total RNA was extracted with the TRIzol reagent (Invitrogen). The RNA quality control parameters were A260/A280 ratio >1.8, A260/A230 ratio $>2.0$ and RIN (RNA integrity number) value (Schroeder et al. 2006) >7.0. The TruSeq RNA sample preparation kit (Illumina) was used to generate RNA-seq libraries following the manufacturer's protocol. High-throughput sequencing was conducted on the Illumina HiSeq 2500 system. Raw data were aligned to mouse genome (UCSC mm9) using TopHat with default options (Trapnell et al. 2009). The aligned reads were then provided as an input for the HTSeq count (Anders et al. 2015) to calculate read counts for each gene in the RefSeq database release 77 (O'Leary et al. 2016). Differential expression analysis was identified using the DESeq package (Anders \& Huber 2010). Differentially expressed genes were chosen according to the criteria of fold-change $>2$ and adjusted $P$ value $<0.01$.

\section{Validation by quantitative RT-PCR}

Total RNA was extracted with TRIzol reagent (Invitrogen). The cDNA was synthesized using the PrimeScript reverse transcriptase reagent kit (TaKaRa). Quantitative RT-PCR was carried out using the SYBR Premix Ex Taq kit (TaKaRa) on the Rotor-Gene 3000A system (Corbett Research). The Rpl7 served as the reference gene for normalization. Primer sequences were listed in Supplementary Table 1 (see section on supplementary data given at the end of this article).

\section{Gene ontology (GO) analysis}

GO analysis was performed by using the DAVID tool (Huang et al. 2007). GO terms with a gene count $<10$ were excluded from further analysis. The significance cutoff for adjusted $P$ value (the false discovery rate method) was set at 0.01. Redundant GO terms were removed manually.

\section{Gene network analysis}

The gene network was generated using the STRING database, v10.0 (Szklarczyk et al. 2015). The minimum combined score was set to 0.9. The Cytoscape software was applied for visualization and analysis of the gene network. The degree distribution was analyzed using the Cytoscape plugin Network Analyzer (Assenov et al. 2008). The degree threshold value for hub genes was the mean plus two standard deviations.

\section{Analysis of transcription factor-binding sites (TFBS)}

The putative promoter regions ( $1 \mathrm{~kb}$ upstream of transcription start site) were retrieved from the UCSC genome browser (Speir et al. 2016). The TESS software, version 6.0 (Schug 2008), was used to search for matches of position-weigh matrices (PWM) available in the TRANSFAC database (Wingender et al. 1996). The cutoff value for relative score was set at 0.9. Using all genes in the genome as the background, a hypergeometric test with Benjamini-Hochberg multiple test correction was conducted using in-house PERL scripts. In the end, adjusted $P$ value $<0.01$ was used as the significance threshold to identify enriched transcription factors.

\section{Statistical analysis}

Statistical analyses were performed in MATLAB 7.5 (MathWorks). Statistical significance of paired $t$-test was set at $P$ value $<0.05$.

\section{Results}

\section{Transcriptomic differences at anti-mesometrial region compared to mesometrial region}

To assess the molecular bases of regional differences in decidualization, we performed RNA-seq on antimesometrial $(A M)$ region and mesometrial $(M)$ region of mouse uterus on day 8 of pregnancy, with three biological replicates respectively (Fig. 1A). Differentially expressed genes were identified with the DESeq package using the following criteria: fold change $>2$ and adjusted $P$ value $<0.01$. As a result, 811 genes were upregulated and 612 genes were downregulated in the AM region compared to those in the $M$ region respectively (Fig. 1B) (Supplementary Table 2). To validate the RNA-seq data, a total of 16 genes with various fold changes (nonchanged: Alpl; upregulated: Ldhb, Rrm2, Hand2, Dio3, Cryab, Prl8a2, Prl3c1 and Prss29; downregulated: Gzma, Wnt6, Wnt5a, Nr2f1, Fn1, Vim and Ldha) were selected and validated by quantitative RT-PCR (qRT$P C R)$. Although there was variation in fold changes between qRT-qPCR and RNA-seq, the expression patterns were coincident between these two techniques (Fig. 1C), indicating a high quality of our RNA-seq data. 
A

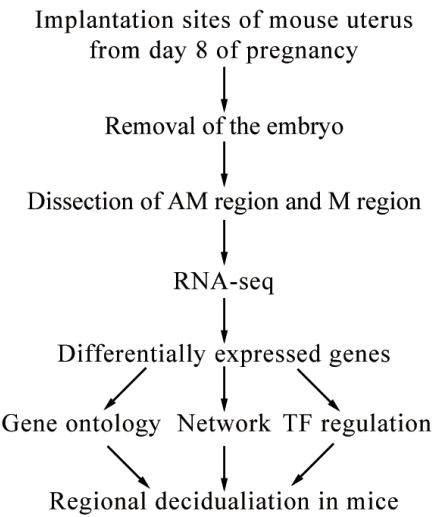

B

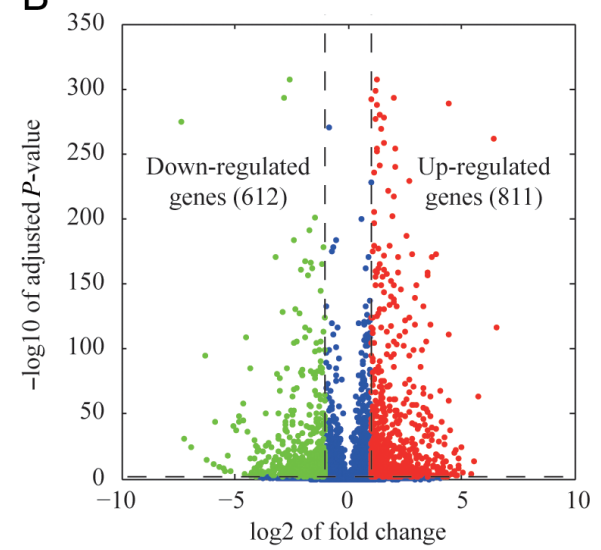

C

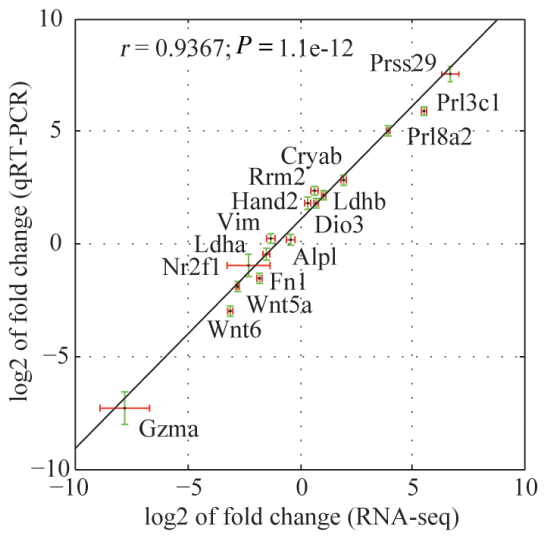

Figure 1 Transcriptomic analysis of regional differences in decidualization of the mouse uterus. (A) A flowchart of the experimental design. M, mesometrial side; AM, anti-mesometrial side; TF, transcription factor. (B) Volcano plot for the comparison between AM and M samples. The cutoff values fold change $>2$ and adjusted $P$ value $<0.01$ were utilized to identify differentially expressed genes. Non-changed genes were shown in blue color. Red color is indicative of upregulated genes and green is indicative of downregulated genes. (C) Validation of selected genes identified by RNA-Seq using qRT-PCR. Fold-change values determined by both RNA-seq and qRT-PCR were presented as the mean \pm S.D. $n=3$.

\section{Characterizing differentially expressed genes by gene ontology analysis}

Gene ontology (GO) analysis of differentially expressed genes was performed to infer the functional consequences of their regional expression pattern. Over-represented GO terms, grouped in the three categories, biological process (BP), cellular component (CC) and molecular function (MF), were identified using the DAVID tool. The significance cutoff for FDR was set at 0.01 . For upregulated genes, there were a total of 18 over-represented terms (Fig. 2A). In the BP category, 4 $\mathrm{GO}$ terms were significantly over-represented, namely ion transport, lipid catabolic process, cell adhesion and epithelial cell differentiation. The over-represented GO terms under the CC category were extracellular region, plasma membrane and cell-cell junction. With respect to the MF category, the over-represented GO terms were calcium ion binding, substrate-specific channel activity, alkali metal ion binding, channel activity, passive transmembrane transporter activity, serine hydrolase activity, serine-type endopeptidase activity, phosphoric diester hydrolase activity, serine-type peptidase activity, ion binding and phospholipase activity. In contrast, for downregulated genes, a total of $16 \mathrm{GO}$ terms were overrepresented (Fig. 2B), including lymphocyte activation, immune response, lymphocyte differentiation, cell adhesion, regulation of cytokine production, immune effector process, cell morphogenesis, hemopoiesis and cell morphogenesis involved in differentiation in the BP category, plasma membrane, proteinaceous extracellular matrix, extracellular region, cell surface and external side of plasma membrane under the CC category, and endopeptidase activity and calcium ion binding from the MF category. Only a small portion of over-represented GO terms (cell adhesion, plasma membrane, extracellular region and calcium ion binding) were shared by both upregulated genes and downregulated genes, indicating that genes selectively enriched in these two compartments were functionally different.

\section{Identification of hub genes by network analysis}

To develop a thorough picture of differentially expressed genes at the systems level, gene network analysis was performed using integrative gene-gene interaction data from the STRING database. The reconstructed gene network for upregulated genes contained 56 genes and 184 gene-gene interactions (Fig. 3A), whereas the reconstructed gene network for downregulated genes consisted of 62 genes and 117 gene-gene interactions (Fig. 3B). Degree distribution analysis showed that both networks followed a power-law distribution and therefore belonged to scale-free small world networks (Barabasi \& Oltvai 2004). Small world networks have the particular feature that some nodes, known as hub genes, are highly connected compared with others. Using a defined threshold value, we identified 4 hub genes (Plcb1, Kng1, Kng2 and Rhoc) for the network of upregulated genes and 2 hub genes (Vav1 and Gng2) for the network of downregulated genes. These genes represent functionally important genes due to their key positions in the networks and thus deserve further investigation.

\section{Regulatory mechanisms revealed by the analysis of transcription factor-binding sites}

Transcription factors are key regulators of gene expression. We extracted proximal promoter sequences 

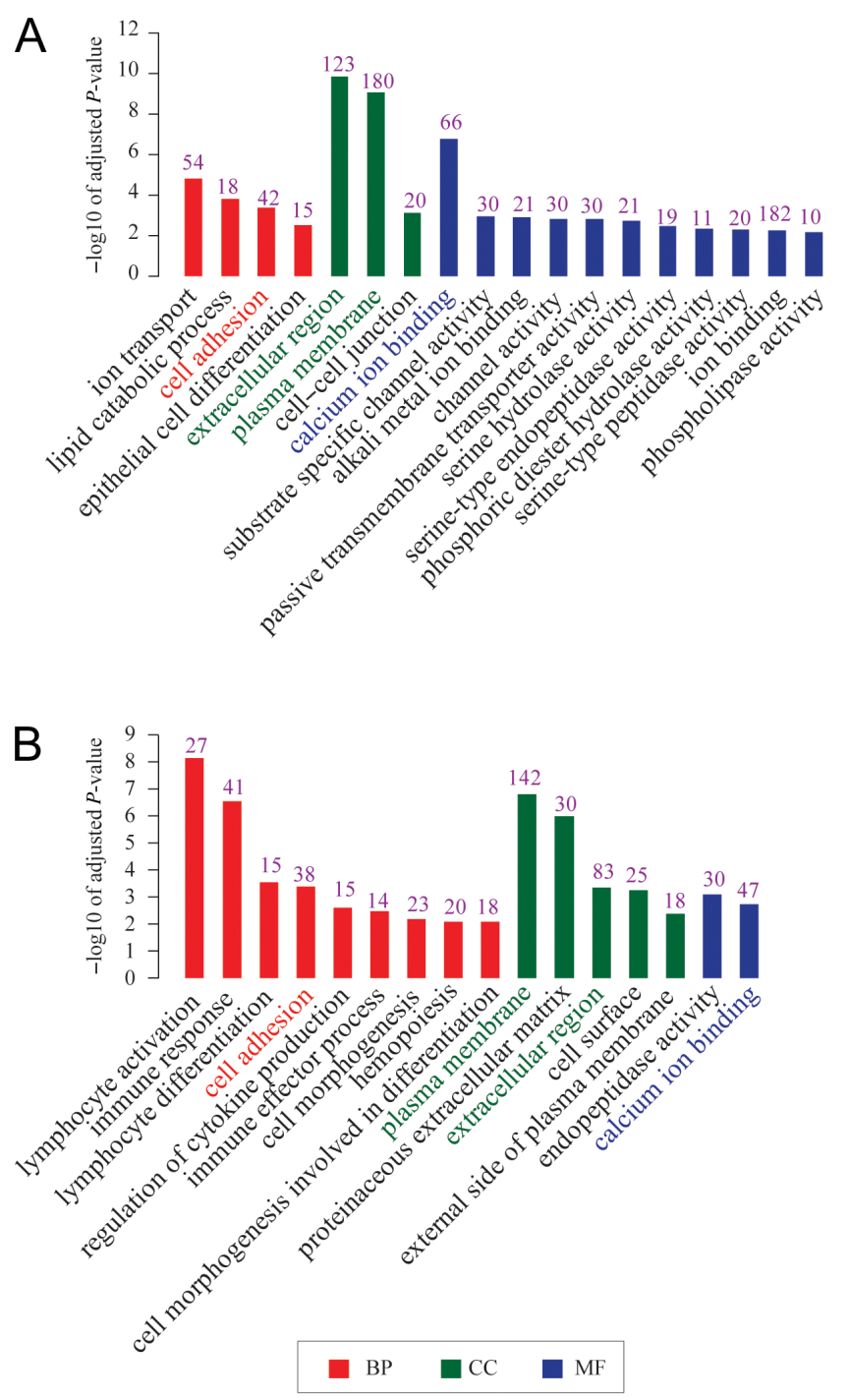

Figure 2 Gene ontology (GO) analysis of differentially expressed genes. (A) Significant GO terms associated with upregulated genes. (B) Significant GO terms associated with downregulated genes. The enrichment test was performed by using the DAVID tool and the significance cutoff for adjusted $P$ value was set at 0.01 . The number on the top of each bar is gene count. GO terms are arranged in 3 categories: biological process (BP), cellular component (CC) and molecular function (MF) respectively.

for all differentially expressed genes. Transcription factor-binding sites were predicted using the TESS software configured with position-weigh matrices (PWM) from the TRANSFAC database. Upregulated genes and downregulated genes were separately tested for over-representation of transcription factor-binding sites. For upregulated genes, the binding sites of SREBP-1 (M00749), NF-kappaB (M00052), STAT6 (M00500), MAZ (M00649), CAC-BP (M00720) and LBP-1 (M00644) were significantly over-represented. For downregulated genes, only the SREBP-1 (M00749)-binding sites were significantly over-represented (Fig. 4). This analysis provides clues to the regulatory mechanisms underlying regional decidualization of mouse uterus.

\section{Global comparison between natural pregnancy and artificial decidualization}

In mice, hormonally primed uterus can be stimulated by mechanical means (e.g. sesame oil) to trigger decidualization in the absence of an embryo (Ledford et al. 1976). The mechanically decidualized endometrium, known as the deciduoma, is morphologically similar to decidualization in natural pregnancy. To determine whether the observed regional decidualization in mouse uterus during natural pregnancy is dependent on the embryo, AM and M samples from artificially decidualized uterus were collected and subjected to RNA-seq. Whole-transcriptome Pearson correlation analysis demonstrated that natural pregnancy and artificial decidualization were highly similar for both $\mathrm{AM}$ and $\mathrm{M}$ samples (Fig. 5A). Differentially expressed genes were identified using the same criteria as described previously, leading to the discovery of 1206 upregulated genes and 1122 downregulated genes in AM region compared to those in $\mathrm{M}$ region (Supplementary Table 3). Among these genes, 578 upregulated genes and 517 downregulated genes were shared with natural pregnancy (Fig. 5B). To gain a systematic comparison, unsupervised hierarchical clustering analysis was conducted on the union of differentially expressed genes identified in natural pregnancy plus differentially expressed genes identified in artificial decidualization (Fig. 5C). The branching pattern illustrated in the dendrogram confirmed the transcriptomic similarity between natural pregnancy and artificial decidualization. In addition, results from qRT-PCR performed on a selected set of genes confirmed the consistency of gene expression pattern between natural pregnancy and artificial decidualization model (Supplementary Fig. 1). Taken together, these results suggest that regional decidualization also occurs in artificial decidualization in a similar way to natural pregnancy. Nevertheless, we did identify a small set of genes that were inconsistently expressed between artificial decidualization and natural pregnancy (Fig. 6A) (Supplementary Table 4). Two genes (Prap1 and Spint2) that were upregulated in natural pregnancy but downregulated in artificial decidualization model were validated using qRT-PCR (Fig. 6B). These genes were likely under the direct or indirect control of embryoderived paracrine signals (Kashiwagi et al. 2007, McConaha et al. 2011).

\section{Discussion}

Although regional differences in mouse decidualization have been recognized for decades, the molecular mechanisms remain understudied. The present study 

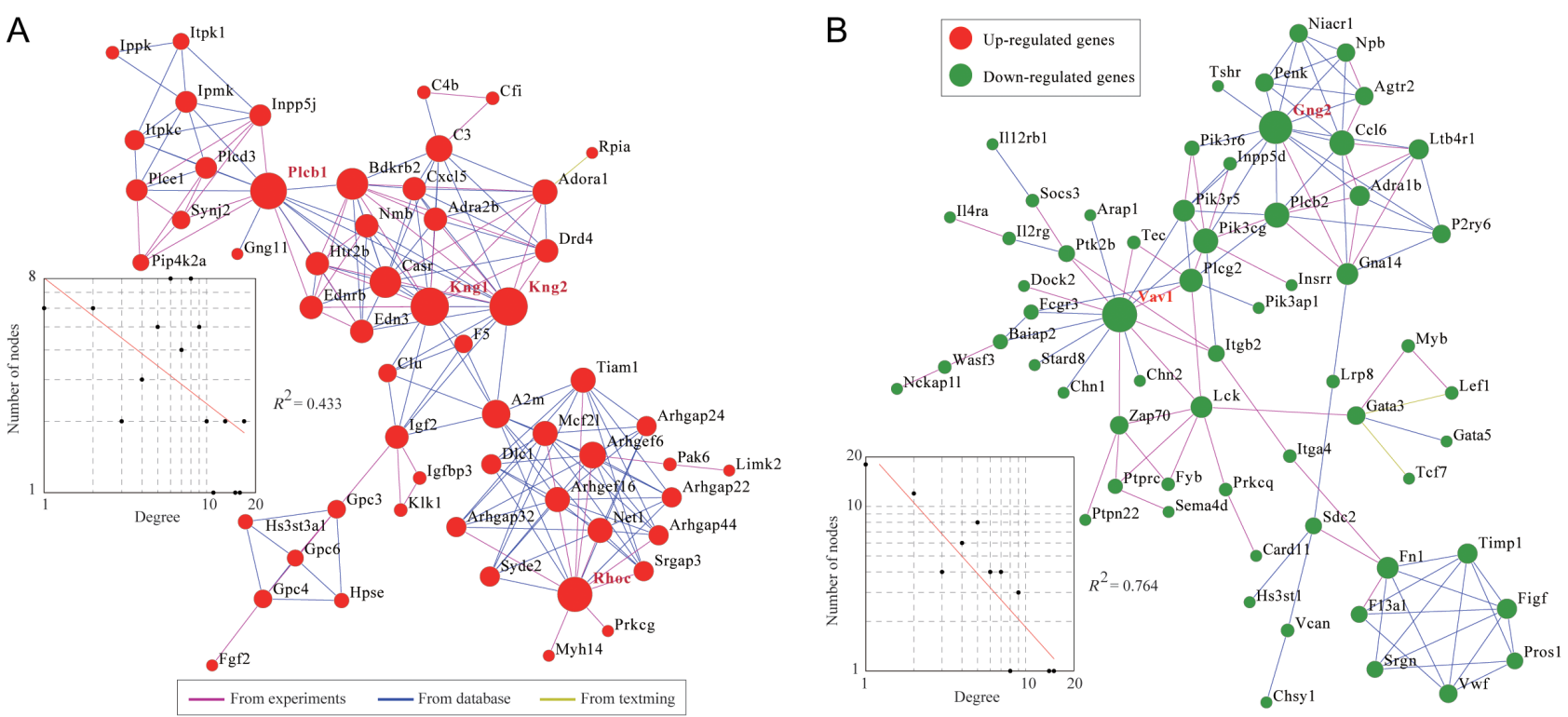

Figure 3 Gene network analysis of differentially expressed genes. (A) The network underlying upregulated genes. (B) The network underlying downregulated genes. This analysis was performed by using the STRING software. Nodes represent genes and edges represent gene-gene interactions. The diameter of each node is proportional to its degree value. This graph was generated using the Cytoscape software. The degree distribution of the network is shown as an inset. The degree distribution follows a power law distribution.

reports the first application of RNA-seq to characterize the transcriptomic differences between the antimesometrial (AM) region and the mesometrial $(\mathrm{M})$ region of mouse uterus on day 8 of pregnancy. Implantation occurs on day 5 of pregnancy. The embryo does not

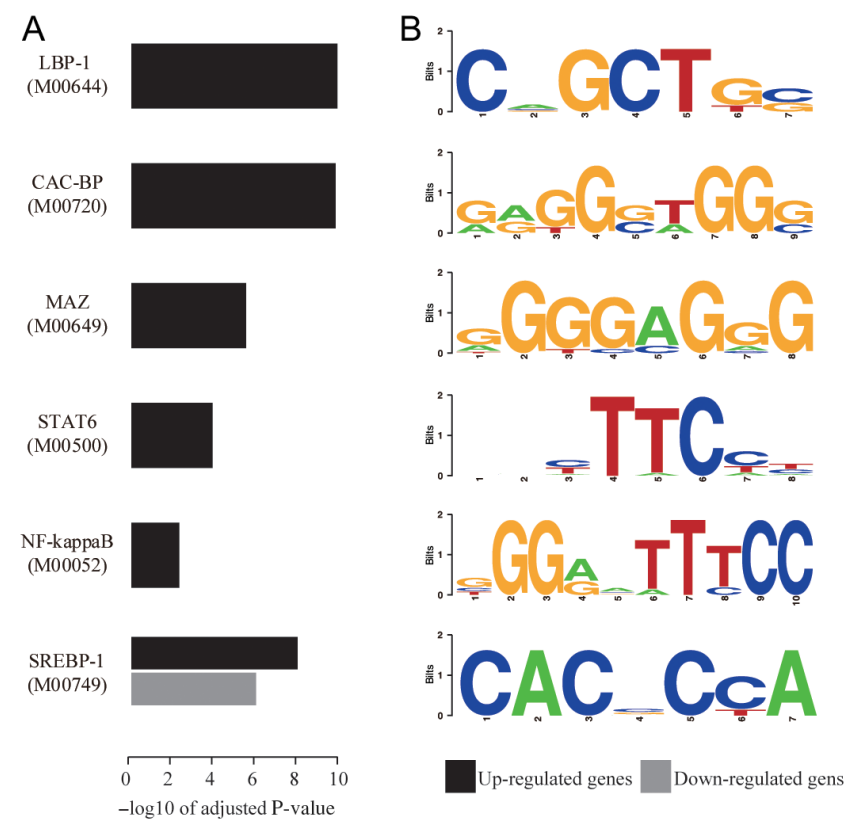

Figure 4 Analysis of transcription factor-binding sites.

(A) Transcription factor analysis using the upregulated genes and downregulated genes respectively. (B) The sequence logo display for all transcription factors whose binding sites are significantly enriched. invade into the uterus until day 6 . On day 8 , the whole uterus, from $\mathrm{AM}$ region to $\mathrm{M}$ region, is in contact with the embryo. In response to embryo, the surrounding decidua is well developed. Placentation initiates on day 8 . Therefore, we chose day 8 in this study as a crucial point for decidualization and placentation. We identified a total of 1423 differentially expressed genes. Quantitative RT-PCR (qRT-PCR) analysis demonstrated that the expression trend of selected genes was consistent with RNA-seq. This is in line with previous studies indicating that RNA-seq is a reliable and reproducible method to evaluate RNA expression (Mortazavi et al. 2008). Throughout the study, the CD-1 mouse strain was used. Compared to inbred mice, the outbred CD-1 mouse is easy to maintain and handle and reproduce quickly. Most importantly, the implantation rate is very stable between experiments. In our previous study, we demonstrated that female fertility in mice is highly variable among inbred strains (Liu et al. 2016). Therefore, strain-dependent differences in uterine gene expression may exist. However, the extent of differences has not been reported in literature yet.

Previously, using text mining, we compiled a list of 287 known genes associated with mouse decidualization and 286 genes associated with mouse decidualization, with an overlap of 111 genes shared by both species (Liu \& Wang 2015). According to our RNA-seq data, 59 genes were differentially expressed: 33 genes were upregulated and 26 genes were downregulated in AM region compared to those in the $M$ region (shown in Supplementary Table 5). These data suggest that regional differences in gene expression are relatively common 
A
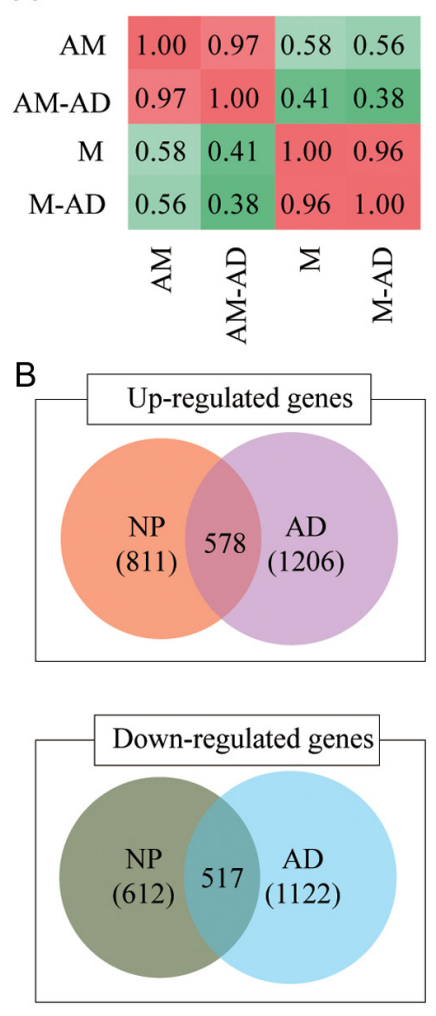

Figure 5 Global comparison of the gene expression changes in artificial decidualization (AD) model against natural pregnancy (NP). (A) Mean Pearson correlation coefficients comparing the global gene expression changes between AD and NP. (B) Venn diagrams showing the number of differentially expressed genes using the criteria: fold change $>2$ and adjusted $P$ value $<0.01$. (C) Cluster dendrogram of the union gene set of differentially expressed genes in NP and differentially expressed genes in AD. The Pearson correlation distance measure and the average linkage clustering algorithm were used. The plot was generated by using bioinformatics toolbox 3.3 of MATLAB (MathWorks).

during mouse decidualization. Prolactin-related protein (Prl8a2) and alkaline phosphatase (Alpl) are two wellknown biomarkers of decidualization in mice (Finn \& Hinchliffe 1964, Orwig et al. 1997, Soares et al. 1998, Li et al. 2007). Interestingly, although Alpl was evenly expressed, Prl8a2 was approximately 16-folds higher in AM region compared to that in $\mathrm{M}$ region. This result was confirmed by qRT-PCR. Thus, Alpl is a mutual biomarker for decidualization in $\mathrm{AM}$ region and $\mathrm{M}$ region, whereas Prl8a2 is a selective biomarker for decidualization in AM region.

Because of the anatomy differences and eccentric embryo implantation in the mouse (Lee \& DeMayo 2004), our data cannot be extrapolated to the human in a straightforward way. Unlike the case of mouse, decidualization is initiated spontaneously in the secretory phase of menstrual cycle in human. If embryo undergoes implantation, decidualization continues; otherwise, menstruation occurs. Previously, a study has demonstrated that perivascular endometrial stromal cells are programmed to mount a distinctive decidual response (Murakami et al. 2014). Given that the M region of mouse uterus is enriched in blood vessels, we suspected that the majority of human perivascular endometrial stromal cells may be similar to the AM region of mouse uterus, whereas the perivascular endometrial stromal cells are equivalent to the $\mathrm{M}$ region of mouse uterus. Further studies are needed to verify this hypothesis.

Gene ontology (GO) analysis provided us important clues to the functional consequences of differentially expressed genes. As expected, upregulated genes were generally involved in cell metabolism and differentiation, whereas downregulated genes were associated with lymphocyte themes and immune response. When trophoblast invasion triggers by inflammatory response in the AM side, immune cells may be recruited into uterus from the vessel-enriched $M$ side. Uterine dendritic cells (DC) are crucial for decidua formation and placental development (Krey et al. 2008, Plaks et al. 2008). Uterine regulatory $T$ cells (Treg) contribute to the maintenance of tolerance to paternal antigens in fetal cells (Samstein et al. 2012). Uterine natural killer cells (uNK) and macrophages participate in the reconstruction of uterine vasculature, which is fundamental to placentation and gestation maintenance (Nagamatsu \& Schust 2010, Ratsep et al. 2015). Our results supported the theory that decidualization in the AM region is involved in the accommodation of embryo implantation, and decidualization in the $M$ region contributes to the preparation of placentation (Dey et al. 2004).

Network analysis was performed to identify hub genes for upregulated genes and downregulated genes. The 4 hub genes for network of upregulated genes were Plcb1 (phospholipase C beta 1), Kng1 (kininogen 1), Kng2 (kininogen 2) and Rhoc (ras homolog gene family member C). The 2 hub genes for network of downregulated genes were Vav1 (vav 1 oncogene) and Gng2 (guanine nucleotide-binding protein gamma 2). Plcb1, Rhoc, Vav1 and Gng2 are related to G proteins. Kng1 and Kng2 have multiple effects in the vascular system (Sharma 2006). These hub genes are likely more important than other genes due to their key positions in the network.

Transcription factors are key regulators of gene expression. Interestingly, we found that SREBP-1 (sterol regulatory element-binding protein 1) is a commonly over-represented transcription factor for both upregulated and downregulated genes. SREBP-1 belongs to a family of basic helix-loop-helix-leucine zipper (bHLHLZ) transcription factors (Yokoyama et al. 1993). Known target genes of SREBP-1 are involved in cholesterol biosynthesis and transport (Brown \& Goldstein 1997, 1999). Additionally, we found that 

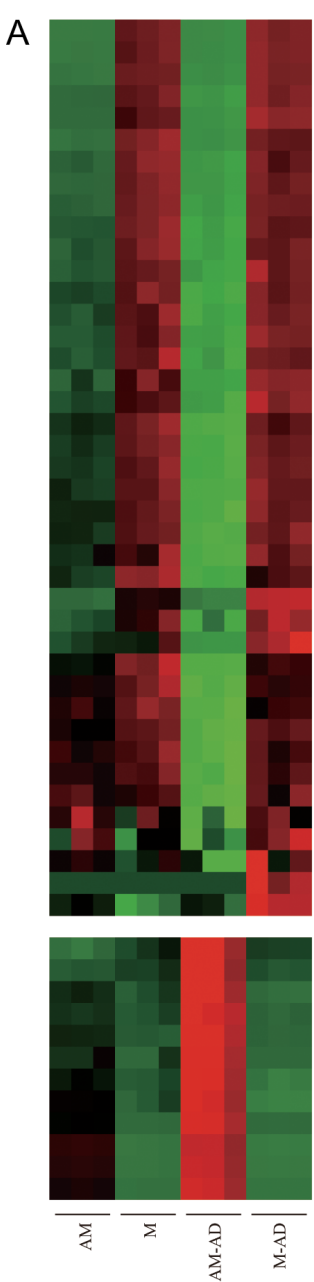

wingless-related MMTV integration site $5 \mathrm{~A}$ (Wnt5a)

elastin (Eln)
protein tyrosine phosphatase receptor type $\mathrm{C}$ (Ptprc) CD248 antigen, endosialin (Cd248) spondin 2 extracellular matrix protein (Spon2) collagen type XIV alpha 1 (Col14a1) centromere protein $\mathrm{F}$ (Cenpf) myosin heavy polypeptide 10 non-muscle (Myh10) topoisomerase (DNA) II alpha (Top2a) matrilin 2 (Matn2)

cancer susceptibility candidate 5 (Casc5) cancer susceptibility candidate 5 (Casc5) Crotein regulator of cytokinesis 1 (Prc1) C-type lectin domain family 11 , member sema domain (semaphorin) 4A (Sema4a) cyclin-dependent (Nfix)

(Cdk1)

A member 5 (Scara5)

Rho guanine nucleotide exchange factor 25 (Arhgef25)

solute carrier family 1 member 5 (Slcla5)

anillin actin binding protein (An

tropomyosin $1 \mathrm{alph}$
dynamin 1 (Dnm1)

insulin-like growth factor binding protein 6 (Igfbp6) scavenger receptor class $\mathrm{F}$ member 2 (Scarf2) activated leukocyte cell adhesion molecule (Alcam) 1-acylglycerol-3-phosphate O-acyltransferase 4 (Agpat4) arylsulfatase J (Arsj)

low density lipoprotein receptor-related protein 3 (Lrp3) calcium channel voltage-dependent $\mathrm{T}$ type alpha $1 \mathrm{H}$ (Cacnalh) carbonic anhydrase 2 (Car2)

lymphocyte specific 1 (Lsp1)

cystin 1 (Cys1)

transmembrane protein $119($ Tmem119)

cingulin (Cgn)

latent transforming growth factor beta binding protein 4 (Ltbp4) keratin 18 (Krt18)

homeobox B4 (Hoxb4)

RIKEN cDNA 3010003L21 gene (3010003L21Rik)

toll-like receptor 2 (Tlr2)

uncharacterized LOC102638061 (LOC102638061)

insulin-like growth factor binding protein 5 (Igfbp5)

umor necrosis factor receptor superfamily, member 21 (Tnfrsf21) colony stimulating factor 1 receptor $(\mathrm{Csfl} r)$

lectin galactose binding soluble 3 (Lgals3)

solute carrier family 39 (zinc transporter), member 2 (Slc39a2)

protein $\mathrm{C}$ receptor endothelial (Procr)

$\mathrm{T}$ cell immunoglobulin and mucin domain containing 2 (Timd2)

HtrA serine peptidase 3 (Htra3)

Ras association (RalGDS/AF-6) domain family member 8 (Rassf8) endogenous retroviral sequence 3 (Erv3)

prosaposin (Psap)
prolactin family 8 subfamily a member 2 (Prl8a2)

ipase endothelial (Lipg)

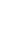

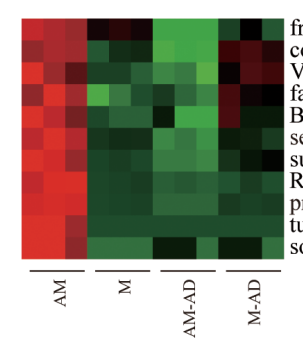

frizzled homolog 10 (Drosophila) (Fzd10) colony stimulating factor 1 (macrophage) (Csfl) $V$-set domain containing $\mathrm{T}$ cell activation inhibitor 1 (Vtcn1) family with sequence similarity 213 member A (Fam 213a) $\mathrm{B}$ cell linker (Blnk) serine protease inhibitor, Kunitz type 2 (Spint2) suppression of tumorigenicity 14 (colon carcinoma) (St14) RIKEN cDNA 5330417C22 gene (5330417C22Rik) proline-rich acidic protein 1 (Prap 1) (Prapl) solute carrier family 5 member 9 (Slc5a9)

Low
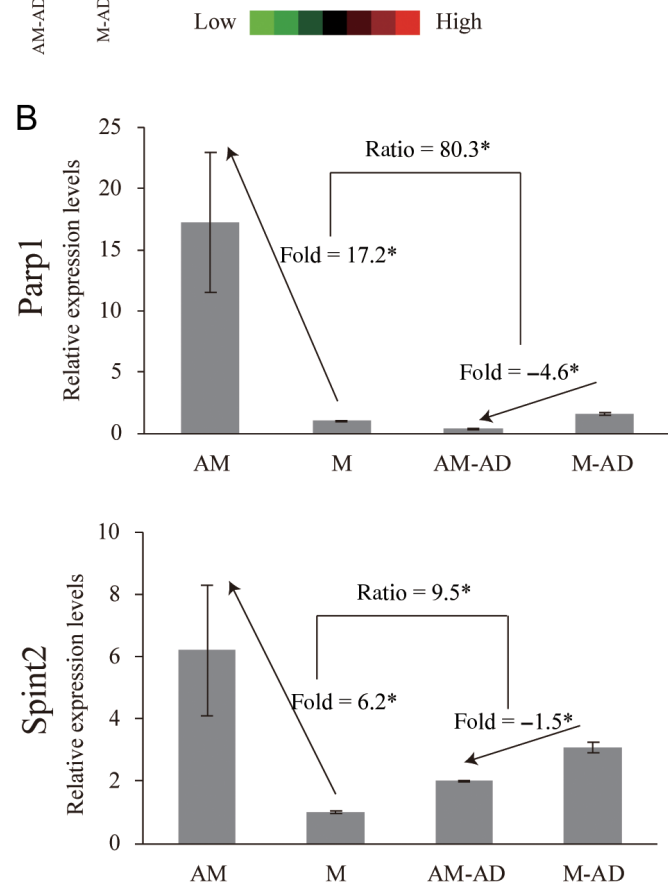

Figure 6 Identification of inconsistently expressed genes between artificial decidualization (AD) and natural pregnancy (NP). (A) Heatmap of inconsistently expressed genes. Candidate genes were selected based on the following criteria: ratio $>2$ and adjusted $P$-value $<0.01$.

(B) Validation of two representative genes by qRT-PCR. Data were presented as the mean \pm S.D. $n=3$. ${ }^{*} P<0.05$.

the binding sites of NF-kappaB, STAT6, MAZ, CAC-BP and LBP-1 were significantly over-represented in the upregulated genes. NF-kappaB (nuclear factor kappalight-chain-enhancer of activated B cells) is a protein complex that controls immune response, cytokine production and cell survival (Baldwin 1996). NF-kappaB is activated at the implantation site of the mouse uterus during early pregnancy (Nakamura et al. 2004). In human endometrial stromal cells, activated NF-kappaB protects cells from apoptosis during decidualization (Fluhr et al. 2013). STAT6 (signal transducer and activator of transcription 6) and its family member STAT3 share the same binding motif (Mascareno et al. 1998). STAT3 is a key regulator of mouse decidualization. Conditional ablation of STAT3 in uterus results in a failure of decidulization reaction (Lee et al. 2013). CAC-BP (CAC-binding protein) binds CAC box and regulates gene expression in a cis manner. MAZ (Myc-associated zinc finger protein) is a transcription factor with dual roles in transcription initiation and termination. One target of MAZ is MYC ( $\mathrm{v}$-myc avian myelocytomatosis viral oncogene homolog) (Bossone et al. 1992). LBP-1 (upstream binding protein 1) is a member of the NTF (neurogenic element-binding transcription factor) family of transcription factors. Currently, little is known about the role of SREBP-1, CAC-BP, MAZ and LBP-1 in regulating uterine gene expression. Hence, these four transcription factors deserve further investigation.

Is the observed regional decidualization in mouse uterus during natural pregnancy dependent on the embryo? To answer this question, we used the artificial decidualization model, in which the sesame oil was used as a mimic of the embryo (Ledford et al. 1976). Whole-transcriptome Pearson correlation analysis revealed that regional decidualization also occurs in artificial decidualization. In artificial decidualization model, we identified 1206 upregulated genes and 1122 downregulated genes. Among these genes, 578 upregulated genes and 517 downregulated genes were shared with natural pregnancy. Results from 
qRT-PCR performed on a selected set of genes confirmed the consistency of gene expression pattern between natural pregnancy and artificial decidualization model. Nevertheless, we did identify a small set of genes that were inconsistently expressed between artificial decidualization and natural pregnancy. For example, Prap1 and Spint2 were upregulated in natural pregnancy but downregulated in artificial decidualization model. The regulatory mechanism and functional consequence for these genes are unknown.

In conclusion, in the present study, using RNA-seq, we compared the transcriptomic differences between the $\mathrm{AM}$ region and the $\mathrm{M}$ region of mouse uterus on day 8 of pregnancy. Our study provides a valuable resource for in-depth understanding of the molecular mechanisms underlying regional decidualization in mice.

\section{Supplementary data}

This is linked to the online version of the paper at http://dx.doi. org/10.1530/REP-16-0486.

\section{Declaration of interest}

The authors declare that there is no conflict of interest that could be perceived as prejudicing the impartiality of the research reported.

\section{Funding}

This work was funded by National Natural Science Foundation of China (grant number 31271602 to Ji-Long Liu). The funder has no role in study design, data collection, analysis or interpretation.

\section{References}

Anders S \& Huber W 2010 Differential expression analysis for sequence count data. Genome Biology 11 R106. (doi:10.1186/gb-2010-1110-r106)

Anders S, Pyl PT \& Huber W 2015 HTSeq - a python framework to work with high-throughput sequencing data. Bioinformatics 31 166-169. (doi:10.1093/bioinformatics/btu638)

Assenov Y, Ramirez F, Schelhorn SE, Lengauer T \& Albrecht M 2008 Computing topological parameters of biological networks. Bioinformatics 24 282-284. (doi:10.1093/bioinformatics/btm554)

Baldwin AS Jr 1996 The NF-kappa B and I kappa B proteins: new discoveries and insights. Annual Review of Immunology 14 649-683. (doi:10.1146/ annurev.immunol.14.1.649)

Barabasi AL \& Oltvai ZN 2004 Network biology: understanding the cell"s functional organization. Nature Reviews Genetics 5 101-113. (doi:10.1038/nrg1272)

Barrientos G, Freitag N, Tirado-Gonzalez I, Unverdorben L, Jeschke U, Thijssen VL \& Blois SM 2014 Involvement of galectin-1 in reproduction: past, present and future. Human Reproduction Update 20 175-193. (doi:10.1093/humupd/dmt040)

Blois SM, Klapp BF \& Barrientos G 2011 Decidualization and angiogenesis in early pregnancy: unravelling the functions of DC and NK cells. Journal of Reproductive Immunology 88 86-92. (doi:10.1016/j.jri.2010.11.002)

Bossone SA, Asselin C, Patel AJ \& Marcu KB 1992 MAZ, a zinc finger protein, binds to C-MYC and C2 gene sequences regulating transcriptional initiation and termination. PNAS 89 7452-7456. (doi:10.1073/ pnas.89.16.7452)

Brown MS \& Goldstein JL 1997 The SREBP pathway: regulation of cholesterol metabolism by proteolysis of a membrane-bound transcription factor. Cell 89 331-340. (doi:10.1016/S0092-8674(00)80213-5)

Brown MS \& Goldstein JL 1999 A proteolytic pathway that controls the cholesterol content of membranes, cells, and blood. PNAS 96 11041-11048. (doi:10.1073/pnas.96.20.11041)

Dey SK, Lim H, Das SK, Reese J, Paria BC, Daikoku T \& Wang H 2004 Molecular cues to implantation. Endocrine Reviews 25 341-373. (doi:10.1210/er.2003-0020)

Finn CA \& Hinchliffe JR 1964 Reaction of the mouse uterus during implantation and deciduoma formation as demonstrated by changes in the distribution of alkaline phosphatase. Journal of Reproduction and Fertility 8 331-338. (doi:10.1530/jrf.0.0080331)

Fluhr H, Spratte J, Bredow M, Heidrich S \& Zygmunt M 2013 Constitutive activity of Erk1/2 and NF-kappaB protects human endometrial stromal cells from death receptor-mediated apoptosis. Reproductive Biology 13 113-121. (doi:10.1016/j.repbio.2013.03.001)

Gellersen B \& Brosens JJ 2014 Cyclic decidualization of the human endometrium in reproductive health and failure. Endocrine Reviews 35 851-905. (doi:10.1210/er.2014-1045)

Huang DW, Sherman BT, Tan Q, Kir J, Liu D, Bryant D, Guo Y, Stephens R, Baseler MW, Lane HC et al. 2007 DAVID Bioinformatics Resources: expanded annotation database and novel algorithms to better extract biology from large gene lists. Nucleic Acids Research 35 W169-W175. (doi:10.1093/nar/gkm415)

Kashiwagi A, DiGirolamo CM, Kanda Y, Niikura Y, Esmon CT, Hansen TR, Shioda T \& Pru JK 2007 The postimplantation embryo differentially regulates endometrial gene expression and decidualization. Endocrinology 148 4173-4184. (doi:10.1210/en.2007-0268)

Krey G, Frank P, Shaikly V, Barrientos G, Cordo-Russo R, Ringel F, Moschansky P, Chernukhin IV, Metodiev M, Fernández N et al. 2008 In vivo dendritic cell depletion reduces breeding efficiency, affecting implantation and early placental development in mice. Journal of Molecular Medicine 86 999-1011. (doi:10.1007/s00109-008-0379-2)

Ledford BE, Rankin JC, Markwald RR \& Baggett B 1976 Biochemical and morphological changes following artificially stimulated decidualization in the mouse uterus. Biology of Reproduction 15 529-535. (doi:10.1095/ biolreprod15.4.529)

Lee KY \& DeMayo FJ 2004 Animal models of implantation. Reproduction 128 679-695. (doi:10.1530/rep.1.00340)

Lee JH, Kim TH, Oh SJ, Yoo JY, Akira S, Ku BJ, Lydon JP \& Jeong JW 2013 Signal transducer and activator of transcription-3 (Stat3) plays a critical role in implantation via progesterone receptor in uterus. FASEB Journal 27 2553-2563. (doi:10.1096/fj.12-225664)

Li Q, Kannan A, Wang W, Demayo FJ, Taylor RN, Bagchi MK \& Bagchi IC 2007 Bone morphogenetic protein 2 functions via a conserved signaling pathway involving wnt 4 to regulate uterine decidualization in the mouse and the human. Journal of Biological Chemistry 282 31725-31732. (doi:10.1074/jbc.M704723200)

Liu JL \& Wang TS 2015 Systematic analysis of the molecular mechanism underlying decidualization using a text mining approach. PLOS ONE 10 e0134585. (doi:10.1371/journal.pone.0134585)

Liu JL, Wang TS \& Zhao M 2016 Genome-wide association mapping for female infertility in inbred mice. G3 6 2929-2935. (doi:10.1534/ g3.116.031575)

Mascareno E, Dhar M \& Siddiqui MA 1998 Signal transduction and activator of transcription (STAT) protein-dependent activation of angiotensinogen promoter: a cellular signal for hypertrophy in cardiac muscle. PNAS 95 5590-5594. (doi:10.1073/pnas.95.10.5590)

McConaha ME, Eckstrum K, An J, Steinle JJ \& Bany BM 2011 Microarray assessment of the influence of the conceptus on gene expression in the mouse uterus during decidualization. Reproduction 141 511-527. (doi:10.1530/REP-10-0358)

Mortazavi A, Williams BA, McCue K, Schaeffer L \& Wold B 2008 Mapping and quantifying mammalian transcriptomes by RNA-Seq. Nature Methods 5 621-628. (doi:10.1038/nmeth.1226)

Murakami K, Lee YH, Lucas ES, Chan YW, Durairaj RP, Takeda S, Moore JD, Tan BK, Quenby S \& Chan JK 2014 Decidualization induces a secretome switch in perivascular niche cells of the human endometrium. Endocrinology 155 4542-4553. (doi:10.1210/en.2014-1370) 
Nagamatsu T \& Schust DJ 2010 The immunomodulatory roles of macrophages at the maternal-fetal interface. Reproductive Sciences 17 209-218. (doi:10.1177/1933719109349962)

Nakamura H, Kimura T, Ogita K, Nakamura T, Takemura M, Shimoya K, Koyama S, Tsujie T, Koyama M \& Murata Y 2004 NF-kappaB activation at implantation window of the mouse uterus. American Journal of Reproductive Immunology 51 16-21. (doi:10.1046/j.87558920.2003.00116.x)

O'Leary NA, Wright MW, Brister JR, Ciufo S, Haddad D, McVeigh R, Rajput B, Robbertse B, Smith-White B \& Ako-Adjei D 2016 Reference sequence (RefSeq) database at NCBI: current status, taxonomic expansion, and functional annotation. Nucleic Acids Research 44 D733-D745. (doi:10.1093/nar/gkv1189)

Orwig KE, Dai G, Rasmussen CA \& Soares MJ 1997 Decidual/trophoblast prolactin-related protein: characterization of gene structure and cellspecific expression. Endocrinology 138 2491-2500. (doi:10.1210/ endo.138.6.5155)

Plaks V, Birnberg T, Berkutzki T, Sela S, BenYashar A, Kalchenko V, Mor G, Keshet E, Dekel N \& Neeman M 2008 Uterine DCs are crucial for decidua formation during embryo implantation in mice. Journal of Clinical Investigation 118 3954-3965. (doi:10.1172/JCI36682)

Ramathal CY, Bagchi IC, Taylor RN \& Bagchi MK 2010 Endometrial decidualization: of mice and men. Seminars in Reproductive Medicine 28 17-26. (doi:10.1055/s-0029-1242989)

Ratsep MT, Felker AM, Kay VR, Tolusso L, Hofmann AP \& Croy BA 2015 Uterine natural killer cells: supervisors of vasculature construction in early decidua basalis. Reproduction 149 R91-R102. (doi:10.1530/REP14-0271)

Samstein RM, Josefowicz SZ, Arvey A, Treuting PM \& Rudensky AY 2012 Extrathymic generation of regulatory $\mathrm{T}$ cells in placental mammals mitigates maternal-fetal conflict. Cell 150 29-38. (doi:10.1016/j. cell.2012.05.031)

Schroeder A, Mueller O, Stocker S, Salowsky R, Leiber M, Gassmann M, Lightfoot S, Menzel W, Granzow M \& Ragg T 2006 The RIN: an RNA integrity number for assigning integrity values to RNA measurements. BMC Molecular Biology 7 3. (doi:10.1186/1471-2199-7-3)

Schug J 2008 Using TESS to predict transcription factor binding sites in DNA sequence. Current Protocols in Bioinformatics Chapter 2 Unit 26. (doi:10.1002/0471250953.bi0206s21)
Sharma JN 2006 Role of tissue kallikrein-kininogen-kinin pathways in the cardiovascular system. Archives of Medical Research 37 299-306. (doi:10.1016/j.arcmed.2005.08.001)

Soares MJ, Muller H, Orwig KE, Peters TJ \& Dai G 1998 The uteroplacental prolactin family and pregnancy. Biology of Reproduction 58 273-284. (doi:10.1095/biolreprod58.2.273)

Speir ML, Zweig AS, Rosenbloom KR, Raney BJ, Paten B, Nejad P, Lee BT, Learned K, Karolchik D, Hinrichs AS et al. 2016 The UCSC genome browser database: 2016 update. Nucleic Acids Research 44 D717D725. (doi:10.1093/nar/gkv1275)

Szklarczyk D, Franceschini A, Wyder S, Forslund K, Heller D, HuertaCepas J, Simonovic M, Roth A, Santos A, Tsafou KP et al. 2015 STRING v10: protein-protein interaction networks, integrated over the tree of life. Nucleic Acids Research 43 D447-D452. (doi:10.1093/nar/gku1003)

Telgmann R \& Gellersen B 1998 Marker genes of decidualization: activation of the decidual prolactin gene. Human Reproduction Update 4 472-479. (doi:10.1093/humupd/4.5.472)

Trapnell C, Pachter L \& Salzberg SL 2009 TopHat: discovering splice junctions with RNA-Seq. Bioinformatics 25 1105-1111. (doi:10.1093/ bioinformatics/btp120)

Wang H \& Dey SK 2006 Roadmap to embryo implantation: clues from mouse models. Human Reproduction Update 7 185-199. (doi:10.1038/ nrg1808)

Wingender E, Dietze P, Karas H \& Knuppel R 1996 TRANSFAC: a database on transcription factors and their DNA binding sites. Nucleic Acids Research 24 238-241. (doi:10.1093/nar/24.1.238)

Yokoyama C, Wang X, Briggs MR, Admon A, Wu J, Hua X, Goldstein JL \& Brown MS 1993 SREBP-1, a basic-helix-loop-helix-leucine zipper protein that controls transcription of the low density lipoprotein receptor gene. Cell 75 187-197. (doi:10.1016/S0092-8674(05)80095-9)

Received 3 September 2016

First decision 3 October 2016

Revised manuscript received 6 February 2017

Accepted 28 February 2017 